ISBN 82-553-0747-8

No. 6

June

1991

Semiparametric estimation

of parametric hazard rates

by

Nils Lid Hjort 
Invited paper presented at the

Advanced Study Workshop on Survival Analysis and Related Topics,

Columbus, Ohio, June 1991

\title{
SEMIPARAMETRIC ESTIMATION OF PARAMETRIC HAZARD RATES
}

\author{
Nils Lid Hjort \\ University of Oslo \\ Department of Mathematics and Statistics \\ P.B. 1053 Blindern \\ N-0316 Oslo, Norway
}

\begin{abstract}
The best known methods for estimating hazard rate functions in survival analysis models are either purely parametric or purely nonparametric. The parametric ones are sometimes too biased while the nonparametric ones are sometimes too variable. There should therefore be scope for methods that somehow try to combine parametric and nonparametric features. In the present paper three semiparametric approaches to hazard rate estimation are presented. The first idea uses a dynamic local likelihood approach to fit the locally most suitable member in a given parametric class of hazard rates. Thus the parametric hazard rate estimate at time $s$ inserts a parameter estimate that also depends on $s$. The second idea is to write the true hazard as a product of an initial parametric estimate times a correction factor, and then estimate this factor nonparametrically using orthogonal expansions. Finally the third idea is Bayesian in flavour and builds a larger nonparametric hazard process prior around a given parametric hazard model. The hazard estimate in this case is the posterior expectation. Properties of the resulting estimators are discussed.
\end{abstract}

KEY WORDS: dynamic likelihood, hazard rate, nonparametric Bayes, orthogonal expansions, semiparametric estimation

\section{Introduction and summary}

This paper concerns semiparametric type methods of estimating hazard rate functions in models for life history data. The best known methods for estimating such hazard rates are those that are either purely parametric or purely nonparametric. The parametric methods are usually biased since parametric models are usually imperfect, and the nonparametric methods often have high estimation variance. There should accordingly be room for methods that somehow lie between the parametric and the nonparametric ones. Such methods could take a possibly crude parametric guess as a starting point and perform some sort of data-dictated corrections on it. One might hope that such methods are better than the nonparametric ones if the 
true hazard is in the vicinity of the parametric model, while not being much worse than the parametric ones if the parametric model is true.

Rather than proposing one particular method and then discussing its merits in full detail, complete with applications and comparisons with previously published estimators, I have chosen in this article to present three new methods, all sharing the characteristic of trying to nonparametrically correct a parametric estimate. Basic motivation and the most important properties are discussed, but without ambitions of providing complete treatments and comparisons.

Although results can be obtained in a more general framework of counting process models we shall mainly be content to illustrate and investigate ideas for the 'random censorship' model, which is the simplest and perhaps most important special case of such models for censored life-time data. It postulates that life-times $X_{1}^{0}, \ldots, X_{n}^{0}$ from a population are i.i.d. with density $f($.$) , cumulative distribution F($.$) , and$ hazard rate function $\alpha($.$) given by$

$$
\alpha(s) d s=\operatorname{Pr}\left\{X_{i}^{0} \in[s, s+d s] \mid X_{i}^{0} \geq s\right\}=\frac{f(s) d s}{F[s, \infty)} .
$$

The life-time $X_{i}^{0}$ may not be directly observed, however, because of a possibly interfering censoring variable $C_{i}$; only $X_{i}=\min \left(X_{i}^{0}, C_{i}\right)$ and the indicator variable $\delta_{i}=I\left\{X_{i}^{0} \leq C_{i}\right\}$ are observed. For simplicity and concreteness we stipulate that the $C_{i}$ 's are independent of the life-times and i.i.d. according to a distribution with cumulative function $G$. In particular the $n$ pairs $\left(X_{i}, \delta_{i}\right)$ are i.i.d. Finally we shall assume that data are obtained on a finite horizon basis, say on $[0, T]$ for a known and finite $T$. This is convenient for some of the martingale convergence theory and is not a practical limitation.

The parametric approach is to postulate that $\alpha(s)=\alpha(s, \theta)$ for a suitable family, indexed by some one- or multi-dimensional $\theta$. Typical examples include the exponential, the Weibull, the simple frailty model with $\alpha(s)=\theta_{1} /\left(1+\theta_{2} s\right)$, the piecewise constant hazard rate model, the Gompertz-Makeham distribution, the gamma, and the log-normal. Properties of the maximum likelihood method for estimating $\theta$ with censored data have been studied by Borgan (1984) and others under the condition that the model is correct, i.e. that there really is some $\theta_{0}$ with $\alpha(s)=\alpha\left(s, \theta_{0}\right)$ on $[0, T]$. In practice the model is never perfect, however, and it is useful to study estimation methods outside model conditions, where the best parameter is to be thought of as being 'least false' or 'most suitable', as opposed to 'true'. The large-sample behaviour of several estimation methods in this wider setting has been explored in Hjort (1986a, 1991a). Some results about this are reviewed in Section 2 and are used in later sections.

In Section 3 a dynamic likelihood approach to parametric hazard rate estimation is presented. It consists of inserting a local parameter estimate $\widehat{\theta}_{n}(s)$ in $\alpha(s, \theta)$ 
at time $s$, where the parameter estimate is obtained using only information on those individuals that have survived up to $s-\frac{1}{2} h$ and what happens to them on $\left[s-\frac{1}{2} h, s+\frac{1}{2} h\right]$. This amounts to a kind of nonparametric parameter smoothing within a given parametric class. Bias and variance properties are studied, and situations are characterised where the new method performs better than the usual nonparametric method, that of smoothing the empirical cumulative hazard.

Section 4 discusses another approach. Its starting point is the expression $\alpha(s)=$ $\alpha_{0}(s) \beta(s)$ with an initial function $\alpha_{0}$. The correction factor $\beta$ can then be estimated using orthogonal expansions. The theory needed is reasonably simple when the $\alpha_{0}$ function is deterministic. But this expansion approach can expect to work best if few terms are needed, i.e. if the correction factor is not too far from being constant. A natural idea is then to use a parametric $\alpha_{0}(s)=\alpha\left(s, \widehat{\theta}_{n}\right)$ as start function. Theory for this more complicated case is also developed.

The third group of methods are Bayesian in character. If one acknowledges uncertainty of one's parametric assumptions one might as well try to model it. Thus one might envision a 'random Weibull model', for example, where there is a nonparametric prior on the space of all hazard functions, centred at the Weibull in some suitable sense. In Section 5 a couple of such semiparametric Bayesian schemes are presented. At least some of the resulting estimators are natural also from a non-Bayesian point of view.

Finally some supplementing results and remarks are offered in Section 6.

\section{Purely nonparametric and purely parametric estimation}

This section introduces some basic notation and reviews properties of the NelsonAalen estimator for the cumulative hazard function in the nonparametric case and of the maximum likelihood estimator in the parametric case. Since our ambition is to go beyond ordinary parametric methods the properties of these must be considered also outside the conditions of the postulated parametric model. The results we give will be used in later sections.

\subsection{NONPARAMETRIC ESTIMATION}

Introduce the counting process $N_{n}(t)=\sum_{i=1}^{n} I\left\{X_{i} \leq t, \delta_{i}=1\right\}$ and the at-risk process $Y_{n}(s)=\sum_{i=1}^{n} I\left\{X_{i} \geq s\right\}$, and form from these the Nelson-Aalen estimator

$$
\widehat{A}_{n}(t)=\int_{0}^{t} \frac{d N_{n}(s)}{Y_{n}(s)}=\sum_{i=1}^{n} \frac{\delta_{i}}{Y_{n}\left(X_{i}\right)} I\left\{X_{i} \leq t\right\}
$$

for the cumulative hazard rate $A(t)=\int_{0}^{t} \alpha(s) d s$. Its properties are best explained using the martingale $M_{n}(t)=N_{n}(t)-\int_{0}^{t} Y_{n}(s) \alpha(s) d s$. Let $y(s)$ be the limit in probability of $\widehat{y}_{n}(s)=Y_{n}(s) / n$, i.e. $y(s)=F[s, \infty) G[s, \infty)$ under present circumstances. 
A basic large-sample property of $M_{n}$ is that $M_{n}(t) / \sqrt{n}$ goes to a Gaußian martingale $V(t)$ with independent increments and noise level $\operatorname{Var} d V(s)=y(s) \alpha(s) d s$, and, more generally, that $\int_{0}^{t} H_{n}(s) d M_{n}(s) / \sqrt{n}$ tends to $\int_{0}^{t} h(s) d V(s)$ in distribution, in cases where $H_{n}($.$) is previsible (its value at s$ is known at $s-$ ) and converges to the determinstic $h($.$) . It follows from these facts that$

$$
\begin{aligned}
\sqrt{n}\left\{d \widehat{A}_{n}(s)-d A(s)\right\} & =\frac{I\left\{Y_{n}(s) \geq 1\right\}}{\widehat{y}_{n}(s)} \frac{d M_{n}(s)}{\sqrt{n}}-I\left\{Y_{n}(s)=0\right\} d A(s) \\
& \doteq_{d} \widehat{y}_{n}(s)^{-1} d M_{n}(s) / \sqrt{n} \rightarrow_{d} y(s)^{-1} d V(s)
\end{aligned}
$$

in the large-sample limit. In particular $d \widehat{A}_{n}(s)$ is very nearly unbiased for $d A(s)$ (the bias goes to zero exponentially fast) and $\sqrt{n}\left\{\widehat{A}_{n}(t)-A(t)\right\}$ tends to the Gaußian martingale $\int_{0}^{t} y(s)^{-1} d V(s)$ with variance $\int_{0}^{t} y(s)^{-1} \alpha(s) d s$. See for example the recent book Andersen et al. (1991, Ch. II) for more details. The usual nonparametric way of estimating the hazard rate itself is to smooth the Nelson-Aalen and take the derivative, see (3.13).

We will also need to work with $Z_{n}(s)=\sqrt{n}\left\{Y_{n}(s) / n-y(s)\right\}$, which goes to a zero mean Gaußian limit process $Z(s)$ with $\operatorname{cov}\{Z(s), Z(t)\}=y(s \vee t)-y(s) y(t)$. There is joint process convergence

$$
\left(d M_{n}(s) / \sqrt{n}, Z_{n}(t)\right) \rightarrow_{d}(d V(s), Z(t))
$$

with $\operatorname{cov}\{d V(s), Z(t)\}$ equal to $-\alpha(s) d s y(t)$ for $s<t$ and zero for $s \geq t$. Details about this can be found in Hjort (1991a, Section 2).

\subsection{PARAmetric estimation}

A parametric model is of the form $\alpha(s)=\alpha(s, \theta)$, where $\theta=\left(\theta_{1}, \ldots, \theta_{p}\right)^{\prime}$ is some $p$ dimensional parameter. The log-likelihood for the observed data can be written $L_{n}(\theta)=\int_{0}^{T}\left\{\log \alpha(s, \theta) d N_{n}(s)-Y_{n}(s) \alpha(s, \theta) d s\right\}$, see for example Andersen et al. (1991, Ch. VI). This defines the maximum likelihood estimator $\widehat{\theta}_{n}$.

To explain the large-sample behaviour of this estimator, let $U_{n}$ be the $p$-vector of first partial derivatives of $n^{-1} L_{n}(\theta)$. Under natural regularity conditions $U_{n}(\theta)$ tends in probability to $u(\theta)=\int_{0}^{T} y(s) \psi(s, \theta)\{\alpha(s)-\alpha(s, \theta)\} d s$, wherein $\psi(s, \theta)=$ $\partial \log \alpha(s, \theta) / \partial \theta$ and $y(s)$ is as above. The maximum likelihood estimator, which solves $U_{n}\left(\hat{\theta}_{n}\right)=0$, converges in probability to the particular parameter value $\theta_{0}$ that solves $u\left(\theta_{0}\right)=0$. We think of this as the 'least false' or 'agnostic' parameter value, and it minimises the distance measure

$$
d[\alpha, \alpha(., \theta)]=\int_{0}^{T} y[\alpha\{\log \alpha-\log \alpha(., \theta)\}-\{\alpha-\alpha(., \theta)\}] d s
$$


between true model and approximating model. This was proved in Hjort (1986a). In Sections 3 and 4 we shall also need the large-sample distribution, and quote the following result from Hjort (1991a). Consider the $p \times p$-matrix $D \psi(s, \theta)=$ $\partial^{2} \log \alpha(s, \theta) / \partial \theta \partial \theta$ and the function $E(s)=\int_{0}^{s} y(t) \psi\left(t, \theta_{0}\right)\left\{\alpha(t)-\alpha\left(t, \theta_{0}\right)\right\} d t$ (in particular $E(0)=E(T)=0)$. Define $p \times p$-matrices

$$
\begin{aligned}
J & =\int_{0}^{T}\left[y(s) \psi\left(s, \theta_{0}\right) \psi\left(s, \theta_{0}\right)^{\prime} \alpha\left(s, \theta_{0}\right)-y(s) D \psi\left(s, \theta_{0}\right)\left\{\alpha(s)-\alpha\left(s, \theta_{0}\right)\right\}\right] d s, \\
K & =\int_{0}^{T}\left[y(s) \psi\left(s, \theta_{0}\right) \psi\left(s, \theta_{0}\right)^{\prime} \alpha(s)+\left\{\psi\left(s, \theta_{0}\right) E(s)^{\prime}+E(s) \psi\left(s, \theta_{0}\right)^{\prime}\right\} \alpha\left(s, \theta_{0}\right)\right] d s .
\end{aligned}
$$

Hjort (1991a) has shown that

$$
\begin{aligned}
\sqrt{n}\left(\widehat{\theta}_{n}-\theta_{0}\right) & \doteq_{d} J^{-1} \int_{0}^{T} \psi\left(s, \theta_{0}\right)\left[d M_{n}(s) / \sqrt{n}+Z_{n}(s)\left\{\alpha(s)-\alpha\left(s, \theta_{0}\right)\right\} d s\right] \\
& \rightarrow_{d} J^{-1} \int_{0}^{T} \psi\left(s, \theta_{0}\right)\left[d V(s)+Z(s)\left\{\alpha(s)-\alpha\left(s, \theta_{0}\right)\right\} d s\right]
\end{aligned}
$$

and this limit variable is normal with mean zero and covariance matrix $J^{-1} K J^{-1}$.

Note that under model conditions $\alpha(s)$ is indeed equal to $\alpha\left(s, \theta_{0}\right)$, the expressions for $J$ and $K$ simplify and become equal, and we have the more familiar-looking limit distribution $N_{p}\left\{0, J^{-1}\right\}$, a result proved by Borgan (1984).

\section{Dynamic likelihood estimation}

This section presents a local likelihood approach to parametric hazard rate estimation. Of course the parametric estimation method of 2.2 works best if the postulated model is adequate, i.e. if there really is a single $\theta_{0}$ that secures $\alpha(s) \doteq \alpha\left(s, \theta_{0}\right)$ throughout $[0, T]$. Otherwise there is modelling bias present and it could for example be advantageous to use different $\theta_{0}$ 's in different sub-intervals. We shall pursue a somewhat more extreme version of this idea, namely to fit a local estimate $\widehat{\theta}_{n}(s)$ for each $s$, and then use $\alpha\left(s, \widehat{\theta}_{n}(s)\right)$ in the end.

\subsection{DYNAMIC LIKELIHOOD}

We shall need some general results about weighted likelihood estimators, from Hjort (1991a, Section 5). Consider $\int_{0}^{T} W_{n}(t)\left\{\log \alpha(t, \theta) d N_{n}(t)-Y_{n}(t) \alpha(t, \theta) d t\right\}$ instead of the ordinary log-likelihood (which uses $W_{n}(t)=1$ ), and let $\widehat{\theta}_{n, w}$ maximise. Assume that $W_{n}(t)$ is previsible and goes in probability to $w(t)$. The first result is that this estimator is consistent for the particular least false parameter value $\theta_{0, w}$ that minimises the distance function

$$
d_{w}[\alpha, \alpha(., \theta)]=\int_{0}^{T} w y[\alpha\{\log \alpha-\log \alpha(., \theta)\}-\{\alpha-\alpha(., \theta)\}] d t
$$


an appropriate generalisation of (2.4). It also solves $\int_{0}^{T} w(t) y(t) \psi(t, \theta)\{\alpha(t)-$ $\alpha(t, \theta)\} d t=0$. Secondly,

$$
\sqrt{n}\left(\widehat{\theta}_{n, w}-\theta_{0, w}\right) \rightarrow_{d} N_{p}\left\{0, J_{w}^{-1} K_{w} J_{w}^{-1}\right\}
$$

where $J_{w}$ and $K_{w}$ are appropriate generalisations of those appearing in Section 2.3. In fact

$$
\begin{aligned}
J_{w} & =\int_{0}^{T} w y\left[\psi_{0} \psi_{0}^{\prime} \alpha_{0}-D \psi_{0}\left(\alpha-\alpha_{0}\right)\right] d t \\
K_{w} & =\int_{0}^{T}\left[w^{2} y \psi_{0} \psi_{0}^{\prime} \alpha+w\left\{\psi_{0} E_{w}+E_{w} \psi_{0}^{\prime}\right\} \alpha_{0}\right] d t
\end{aligned}
$$

in which $E_{w}(t)=\int_{0}^{t} w y \psi_{0}\left(\alpha-\alpha_{0}\right) d s$, and where $\alpha_{0}=\alpha\left(s, \theta_{0, w}\right), \psi_{0}=\psi\left(s, \theta_{0, w}\right)$. Note that both $E_{w}(0)$ and $E_{w}(T)$ are equal to 0 , and that the expressions for $J_{w}$ and $K_{w}$ simplify when the model happens to be correct.

The local or dynamic likelihood estimation idea is to use this apparatus with $W_{n}(t)=w(t)=I\{t \in B\}$, where $B=\left[s-\frac{1}{2} h, s+\frac{1}{2} h\right]$ is a local interval around a given fixed $s$. So let $\widehat{\theta}_{n}(s)$ maximise $\int_{B}\left\{\log \alpha(t, \theta) d N_{n}(t)-Y_{n}(t) \alpha(t, \theta) d t\right\}$. One can show that this is the likelihood based on only those individuals that have survived up to $s-\frac{1}{2} h$ and information about what happens to them in $\left[s-\frac{1}{2} h, s+\frac{1}{2} h\right]$. The resulting dynamic likelihood hazard rate estimator is

$$
\widehat{\alpha}_{n}(s)=\alpha\left(s, \widehat{\theta}_{n}(s)\right) \text {. }
$$

The $\widehat{\theta}_{n}(s)$ estimator aims at the locally most suitable parameter value $\theta_{0}(s)$ that minimises (3.1) with $w=I_{B}$, or, equivalently, solves $\int_{B} y(t) \psi(t, \theta)\{\alpha(t)-$ $\alpha(t, \theta)\} d t=0$. Its large-sample behaviour is indicated by (3.2), which suggests

$$
E \widehat{\theta}_{n}(s) \doteq \theta_{0}(s), \quad \operatorname{VAR} \widehat{\theta}_{n}(s) \doteq J_{s}^{-1} K_{s} J_{s}^{-1} / n,
$$

where $J_{s}$ and $K_{s}$ are as above with $w(t)=I\{t \in B\}$. This transforms into corresponding properties for $\widehat{\alpha}_{n}(s)$ by Taylor expansions and delta-method arguments:

$$
\begin{aligned}
E \alpha\left(s, \widehat{\theta}_{n}(s)\right) & \doteq \alpha\left(s, \theta_{0}(s)\right) \\
\operatorname{Var} \alpha\left(s, \widehat{\theta}_{n}(s)\right) & \doteq \alpha\left(s, \theta_{0}(s)\right)^{2} \psi\left(s, \theta_{0}(s)\right)^{\prime} J_{s}^{-1} K_{s} J_{s}^{-1} \psi\left(s, \theta_{0}(s)\right) / n
\end{aligned}
$$

These approximations are valid if $h$ is fixed and $n$ is large. But we are also interested in becoming increasingly fine-tuned about the $s \pm \frac{1}{2} h$ interval as $n$ grows. In order to study the bias and variance properties more closely, observe first that if $g(t)$ is a twice differentiable function defined in a neighbourhood of $s$, then 
$\int_{B} g(t) d t \doteq g(s) h+\frac{1}{24} g^{\prime \prime}(s) h^{3}$ by a simple Taylor argument. From this and the defining equation for $\theta_{0}(s)$ we see that

$$
y(s) \psi(s, \theta)\{\alpha(s)-\alpha(s, \theta)\}+\frac{1}{24}(y \psi(., \theta)(\alpha-\alpha(s, \theta)))^{\prime \prime}(s) h^{2} \doteq 0,
$$

for the particular value $\theta=\theta_{0}(s)$, where $(f g h)^{\prime \prime}(s)$ means the second derivative of the $f(s) g(s) h(s)$ function evaluated at $s$. This implies generally that $\alpha\left(s, \theta_{0}(s)\right)=$ $\alpha(s)+O\left(h^{2}\right)$. One can also show from this that

$$
E \alpha\left(s, \widehat{\theta}_{n}(s)\right)=\alpha\left(s, \theta_{0}(s)\right)+O(1 / n)=\alpha(s)+O\left(h^{2}\right)+O(1 / n) .
$$

In order for the bias of the (3.4) estimator to go to zero it is therefore necessary that $h \rightarrow 0$ as $n \rightarrow \infty$.

The multi-parameter case is somewhat unwieldy, and we shall be content to give a bias formula for the case of a one-parameter family $\alpha(s, \theta)$, for which

$$
\alpha\left(s, \theta_{0}(s)\right) \doteq \alpha(s)+\frac{h^{2}}{24}\left[\alpha^{\prime \prime}(s)-\alpha_{0}^{\prime \prime}(s)+2\left\{\alpha^{\prime}(s)-\alpha_{0}^{\prime}(s)\right\}\left\{\frac{y^{\prime}(s)}{y(s)}+\frac{\psi_{0}^{\prime}(s)}{\psi_{0}(s)}\right\}\right]
$$

In this formula $\alpha_{0}^{\prime}(s)$ means the derivative of $\alpha(s, \theta)$ w.r.t. $s$, and then inserted $\theta=\theta_{0}(s)$, and similarly for $\alpha_{0}^{\prime \prime}(s)$ and $\psi_{0}^{\prime}(s)$.

Turning next to the variance matrix, one finds after using the (3.3) expressions and the previously established $O\left(h^{2}\right)$ result for the bias that

$$
J_{s} \doteq y(s) \psi_{0}(s) \psi_{0}(s)^{\prime} \alpha\left(s, \theta_{0}(s)\right) h \text { and } K_{s} \doteq y(s) \psi_{0}(s) \psi_{0}(s)^{\prime} \alpha(s) h,
$$

apart from $O\left(h^{3}\right)$ terms, and writing for simplicity $\psi_{0}(s)$ for $\psi\left(s, \theta_{0}(s)\right)$. Again the multi-parameter case requires more precise expansions, since the inverse of $J_{s}$ is needed and $\psi_{0}(s) \psi_{0}(s)^{\prime}$ has rank 1 . We leave the general case for future work and note here that $\operatorname{Var} \widehat{\theta}_{n}(s) \doteq(n h)^{-1}\left\{y(s) \alpha(s) \psi_{0}(s)^{2}\right\}^{-1}$ in the one-dimensional case, which in its turn implies

$$
\operatorname{Var} \alpha\left(s, \widehat{\theta}_{n}(s)\right) \doteq \frac{1}{n h} \frac{\alpha(s)}{y(s)}
$$

Thus $n h \rightarrow \infty$ is necessary for the variance to go to zero, and this together with $h \rightarrow 0$ suffices for consistency of the (3.4) estimator.

\subsection{SPECIAL CASE: MOVING HAZARD ESTIMATE}

The simplest model to try out is the one having $\alpha(s, \theta)=\theta$, a constant hazard. The local hazard estimate and its limit in probability are

$$
\widehat{\alpha}_{n}(s)=\widehat{\theta}_{n}(s)=\frac{\int_{B} d N_{n}(t)}{\int_{B} Y_{n}(t) d t} \rightarrow_{p} \frac{\int_{B} y(t) \alpha(t) d t}{\int_{B} y(t) d t}=\theta_{0}(s)
$$


again with $B=\left[s-\frac{1}{2} h, s+\frac{1}{2} h\right]$. The estimate is of the type total occurence over total exposure, and the underlying local least false parameter is a local $y$-weighted average of the true hazard rate. By earlier efforts

$$
E \widehat{\alpha}_{n}(s) \doteq \alpha(s)+\frac{h^{2}}{24}\left\{\alpha^{\prime \prime}(s)+2 \alpha^{\prime}(s) \frac{y^{\prime}(s)}{y(s)}\right\} \quad \text { and } \quad \operatorname{Var} \widehat{\alpha}_{n}(s) \doteq \frac{1}{n h} \frac{\alpha(s)}{y(s)}
$$

This can also be verified directly. Some more attention to these details is given in the next subsection.

\subsection{LOCAL KERNEL SMOOTHED LIKELIHOOD}

The local likelihood method of subsections 3.1 and 3.2 can be generalised to kernel smoothed variants. Let $K(u)$ be a symmetric kernel function with support $\left[-\frac{1}{2}, \frac{1}{2}\right]$ and integral 1. Define the local kernel smoothed likelihood estimator $\widehat{\theta}_{n}(s)$ to maximise

$$
\int_{B} h^{-1} K\left(h^{-1}(t-s)\right)\left\{\log \alpha(t, \theta) d N_{n}(t)-Y_{n}(t) \alpha(t, \theta) d t\right\} .
$$

The hazard rate estimator is as in (3.4) with this more general estimate of $\theta$. The previously defined local likelihood estimate corresponds to the special case $K(u)=1$ on $\left[-\frac{1}{2}, \frac{1}{2}\right]$. This choice has perhaps some special appeal since the local likelihood then can be interpreted as the 'real' likelihood for a subgroup of the individuals under study, whereas the current smoothed likelihood is more of a mathematical construction, but turning out to produce estimators with slightly better properties, for good choices of $K(u)$.

Let us illustrate this for the special case where $\alpha(s, \theta)=\theta$. Then

$$
\widehat{\alpha}_{n}(s)=\frac{\int_{B} h^{-1} K\left(h^{-1}(t-s)\right) d N(t)}{\int_{B} h^{-1} K\left(h^{-1}(t-s)\right) Y(t) d t}
$$

a locally weighted occurrence over locally weighted exposure estimate. Write this as

$$
\frac{V}{W} \doteq \frac{v}{w}+\frac{1}{w}(V-v)-\frac{v}{w^{2}}(W-w)+\frac{v}{w^{3}}(W-w)^{2}-\frac{1}{w^{2}}(V-v)(W-w),
$$

say, with

$$
\begin{gathered}
V=\frac{1}{n} \int_{B} K_{h, s}(t)\left\{d M_{n}(t)+Y_{n}(t) \alpha(t) d t\right\} \quad \text { and } \quad v=\int_{B} K_{h, s}(t) y(t) \alpha(t) d t \\
W=\frac{1}{n} \int_{B} K_{h, s}(t) Y_{n}(t) d t \quad \text { and } \quad w=\int_{B} K_{h, s}(t) y(t) d t
\end{gathered}
$$


writing $K_{h, s}(t)=h^{-1} K\left(h^{-1}(t-s)\right)$ (and $s$ is fixed at the moment). Calculations involving Taylor expansions and several arguments resembling those of the previous subsection, as well as the simultaneous convergence result (2.3), lead to

$$
E \widehat{\alpha}_{n}(s)=\frac{v}{w}+O\left(n^{-1}\right) \doteq \alpha(s)+\frac{1}{2} \beta_{K} h^{2}\left\{\alpha^{\prime \prime}(s)+2 \alpha^{\prime}(s) y^{\prime}(s) / y(s)\right\}
$$

$\operatorname{Var} \widehat{\alpha}_{n}(s) \doteq \frac{1}{n} \operatorname{Var}\left\{\frac{1}{w} \int_{B} K_{h, s}\left(d M_{n} / \sqrt{n}+Z_{n} \alpha d t\right)-\frac{v}{w^{2}} \int_{B} K_{h, s} Z_{n} d t\right\} \doteq \frac{\gamma_{K}}{n h} \frac{\alpha(s)}{y(s)}$, where $\beta_{K}=\int u^{2} K(u) d u$ and $\gamma_{K}=\int K(u)^{2} d u$. The fact that $\int u K(u) d u=0$ is used here. When $K(u)$ is uniform we get back (3.9).

Similar results can be obtained for a general one-parameter family $\alpha(s, \theta)$, where $\widehat{\theta}_{n}(s)$ solves $\int_{B} K_{h, s}(t) \psi(t, \theta)\left\{d N_{n}(t)-Y_{n}(t) \alpha(t, \theta) d t\right\}=0$. Details analogous to those above give in the end that

$$
E \alpha\left(s, \widehat{\theta}_{n}(s)\right) \doteq \alpha(s)+\frac{1}{2} h^{2} \beta_{K} b(s) \quad \text { and } \quad \operatorname{Var} \alpha\left(s, \widehat{\theta}_{n}(s)\right) \doteq \frac{\gamma_{K}}{n h} \frac{\alpha(s)}{y(s)}
$$

in which the bias factor is

$$
b(s)=\alpha^{\prime \prime}(s)-\alpha_{0}^{\prime \prime}(s)+2\left\{\alpha^{\prime}(s)-\alpha_{0}^{\prime}(s)\right\}\left\{\frac{y^{\prime}(s)}{y(s)}+\frac{\psi_{0}^{\prime}(s)}{\psi_{0}(s)}\right\},
$$

also appearing in (3.6), which indeed is a special case. Observe that the approximate variance does not depend on the parametric family used.

These results can be compared to similar ones for the more traditional kernel smoothed Nelson-Aalen estimator,

$$
\widetilde{\alpha}_{n}(s)=\int_{B} K_{h, s}(t) d \widehat{A}_{n}(t)
$$

when $K(u)$ is uniform this becomes $\left\{\widehat{A}_{n}\left(s+\frac{1}{2} h\right)-\widehat{A}_{n}\left(s-\frac{1}{2} h\right)\right\} / h$, for example. From the properties of $\widehat{A}_{n}$ reviewed in Section 2 it is not difficult to derive

$$
E \widetilde{\alpha}_{n}(s) \doteq \alpha(s)+\frac{1}{2} \beta_{K} h^{2} \alpha^{\prime \prime}(s) \quad \text { and } \quad \operatorname{Var} \widetilde{\alpha}_{n}(s) \doteq \frac{\gamma_{K}}{n h} \frac{\alpha(s)}{y(s)} .
$$

See also Ramlau-Hansen (1983), Yandell (1983), and Tanner and Wong (1983), who all studied estimators of this type, and Andersen et al. (1991, Ch. IV). It is remarkable that the new estimator $\alpha\left(s, \widehat{\theta}_{n}(s)\right)$ and the traditional one have the same variance and nearly the same bias, when they use the same kernel and the same bandwidth. The new method is better when

$$
\left|\alpha^{\prime \prime}(s)-\alpha_{0}^{\prime \prime}(s)+2\left\{\alpha^{\prime}(s)-\alpha_{0}^{\prime}(s)\right\}\left\{y^{\prime}(s) / y(s)+\psi_{0}^{\prime}(s) / \psi_{0}(s)\right\}\right| \leq\left|\alpha^{\prime \prime}(s)\right|
$$


This can easily happen if the parametric family is only moderately acceptable. For the special case (3.10), for which $\alpha_{0}^{\prime}(s), \alpha_{0}^{\prime \prime}(s)$ and $\psi_{0}^{\prime}(s)$ are absent, the inequality might take place in a region where $\alpha$ is convex and increasing, or concave and decreasing. When there is no censoring $y=\exp (-A)$ and $y^{\prime} / y=-\alpha$, and then the criterion for when (3.10) is better than then the traditional (3.13) becomes $0 \leq \alpha(s) \alpha^{\prime}(s) / \alpha^{\prime \prime}(s) \leq 1$.

\subsection{Choosing the SMoothing Parameter}

We have defined $\widehat{\alpha}_{n}(s)=\alpha\left(s, \widehat{\theta}_{n}(s)\right)$ for given parametric family $\alpha(s, \theta)$ and kernel $K$. The most decisive influence on the estimator is due to the smoothing parameter $h$. By (3.11) the approximate mean squared error is

$$
E\left\{\widehat{\alpha}_{n}(s)-\alpha(s)\right\}^{2} \doteq \frac{1}{4} h^{4} \beta_{K}^{2} b(s)^{2}+\frac{\gamma_{K}}{n h} \frac{\alpha(s)}{y(s)},
$$

which is minimised for

$$
h_{0}(s)=\left\{\frac{\gamma_{K}}{\beta_{K}^{2}} \frac{\alpha(s)}{b(s)^{2}}\right\}^{1 / 5} \frac{1}{\{n y(s)\}^{1 / 5}} .
$$

The resulting minimal mean squared error is $\frac{5}{4}\left(\beta_{K} \gamma_{K}^{2}\right)^{2 / 5} \alpha(s)^{4 / 5} b(s)^{2 / 5} /\{n y(s)\}^{4 / 5}$. Different choices of reasonable kernels give about the same result, but the best choice is the Bartlett-Epanechnikov kernel $K_{0}(u)=\frac{3}{2}\left(1-4 u^{2}\right)$ on $\left[-\frac{1}{2}, \frac{1}{2}\right]$. The $h_{0}$ formula cannot be put to direct use since it depends on the hazard rate itself, but the rate of convergence to zero of mean squared error becomes the optimal $n^{-4 / 5}$ when $h$ is chosen proportional to $n^{-1 / 5}$. The formula indicates that $h$ should be chosen proportional to $Y_{n}(s)^{-1 / 5}$ in practice. One possibility is to use $h_{n}=c Y_{n}(s)^{-1 / 5}$ and try to minimise a global criterion like $E \int_{0}^{T} y(s)^{4 / 5}\left\{\widehat{\alpha}_{n}(s)-\alpha(s)\right\}^{2} d s$ w.r.t. $c$. The result is a local variable kernel smoothed likelihood with

$$
h_{n}=\left\{\frac{\gamma_{K}}{\beta_{K}^{2}} \frac{\int_{0}^{T} \alpha(s) d s}{\int_{0}^{T} b(s)^{2} d s}\right\}^{1 / 5} \frac{1}{Y_{n}(s)^{1 / 5}} .
$$

The first integral can be estimated with $n^{1 / 2}$-precision. Some pilot estimate $\alpha_{\text {pil }}$, like the (3.13) estimator with a somewhat large $h$, can be inserted in the second integral to give a practical algorithm.

Observe that when the rough parametric family $\alpha(s, \theta)$ used is moderately acceptable, then the size of $b(s)$ of (3.12) is small, which suggests using quite a large bandwidth $h$, which in its turn almost amounts to using an ordinary parametric method. And if the parametric model doesn't fit our method resembles the nonparametric Nelson-Aalen smoother (3.13) in performance. One natural but elaborate strategy would be to choose $h=h_{n}(s)$ to be the smallest $h$ for which some convenient goodness of fit criterion rejects the parametric model on $s \pm \frac{1}{2} h$; see Hjort (1990a) for such tests. 
The reasoning that led to (3.15) and (3.16) is also pertinent for the problem of choosing $h$ in the Ramlau-Hansen-Yandell estimator (3.13), since the bias and variance structure are of the same type, only with $b(s)=\alpha^{\prime \prime}(s)$ instead. We also note that there are other ways of obtaining a data-driven $h_{n}(s)$, like cross validation or bootstrapping, but these are not discussed here.

\section{Orthogonal expansions with a parametric start}

In this section another semiparametric method is presented. Methods and results are developed for hazard rate estimators formed by approximating the correction factor $\beta(s)$ in $\alpha(s)=\alpha_{0}(s) \beta(s)$ with a finite linear combination of orthogonal basis functions. The theory is fairly uncomplicated as long as the start function $\alpha_{0}(s)$ is deterministic, say $\alpha_{0}(s)=1$, and indeed resembles known theory for the orthogonal expansion approach to nonparametric density estimation. Further theory is developed in 4.2 to handle the case where the initial function is itself a parametric estimate, say $\alpha_{0}(s)=\alpha\left(s, \widehat{\theta}_{n}\right)$. The methods and some of the results of 4.1 were first presented in Hjort (1985a). Those of 4.2 are new but parallel methods were worked out in Hjort (1986b, Ch. 5) for the somewhat simpler nonparametric density estimation problem.

\subsection{EXPANSIONS WITH A FIXED START}

The basis of the expansion approach is a set of functions $\left\{p_{0}, p_{1}, \ldots\right\}$ on $[0, T]$ that are orthogonal w.r.t. a suitable weight function $w$, that is $\int_{0}^{T} p_{j}(s) p_{l}(s) w(s) d s=$ $\delta_{j, l}=I\{j=l\}$. One usually takes $w$ to be a density so that $p_{0}(s)$ can be chosen as 1 . The most important examples are perhaps the cosine-functions $p_{j}(s)=$ $(2 / T)^{1 / 2} \cos (j \pi s / T)$ and the normalised Legendre polynomials, see Abramowitz and Stegun (1964, Ch. 22). In both cases the uniform weight function $w(s)=1 / T$ is used.

Let the expansion start with a given function $\alpha_{0}(s)$, for example a rough start guess or a constant or simply $\alpha_{0}(s)=1$, and write $\alpha(s)=\alpha_{0}(s) \beta(s)$. The idea is to estimate the correction term $\beta(s)=\alpha(s) / \alpha_{0}(s)$ with an orthogonal expansion of the type $\sum_{j=0}^{m} c(j) p_{j}(s)$. The best approximation, as measured by integrated squared error

$$
\int_{0}^{T}\left\{\beta(s)-\sum_{j=0}^{m} c(j) p_{j}(s)\right\}^{2} w(s) d s=\int_{0}^{T}\left\{\alpha(s)-\alpha_{0}(s) \sum_{j=0}^{m} c(j) p_{j}(s)\right\}^{2} \frac{w(s)}{\alpha_{0}(s)^{2}} d s
$$

takes places when $c(j)=\int_{0}^{T} p_{j}(s) w(s) \beta(s) d s=\int_{0}^{T} p_{j}(s) w(s) \alpha_{0}(s)^{-1} d A(s)$. In fact $\beta=\sum_{j=0}^{\infty} c(j) p_{j}$. The $c(j)$ coefficient is an unknown quantity but a natural estimate is

$$
\widehat{c}(j)=\int_{0}^{T} p_{j}(s) w(s) \alpha_{0}(s)^{-1} d \widehat{A}_{n}(s)=\int_{0}^{T} p_{j}(s) \frac{w(s)}{\alpha_{0}(s)} \frac{d N_{n}(s)}{Y_{n}(s)},
$$


inviting the hazard rate estimator

$$
\widehat{\alpha}_{m}(s)=\alpha_{0}(s) \sum_{j=0}^{m} \widehat{c}(j) p_{j}(s) .
$$

In order to balance bias against variance for $\widehat{\alpha}_{m}$, consider the integrated squared error

$$
\begin{aligned}
\operatorname{ISE}(m) & =\int_{0}^{T}\left\{\widehat{\alpha}_{m}(s)-\alpha(s)\right\}^{2} \alpha_{0}(s)^{-2} w(s) d s \\
& =\int_{0}^{T}\left[\sum_{j=0}^{m}\{\widehat{c}(j)-c(j)\} p_{j}(s)-\sum_{j=m+1}^{\infty} c(j) p_{j}(s)\right]^{2} w(s) d s \\
& =\sum_{j=0}^{m}\{\widehat{c}(j)-c(j)\}^{2}+\sum_{j=m+1}^{\infty} c(j)^{2}=\sum_{j=0}^{\infty} c(j)^{2}-D(m),
\end{aligned}
$$

in which

$$
D(m)=\sum_{j=0}^{m}\left[c(j)^{2}-\{\widehat{c}(j)-c(j)\}^{2}\right] .
$$

We would like to choose the order $m$ so as to make $D(m)$ as large as possible. But $D(m)$ is random and depends on unknown quantities. One natural way to proceed is to calculate $H(m)=E D(m)$ and then estimate the $H(m)$ quantities from data.

It follows from (2.2) that $\widehat{c}(j)$ is very nearly unbiased for $c(j)$ and that

$$
\begin{aligned}
\sqrt{n}\{\widehat{c}(j)-c(j)\} & \doteq_{d} \int_{0}^{T} p_{j}(s) w(s) \alpha_{0}(s)^{-1} \frac{n}{Y_{n}(s)} \frac{d M_{n}(s)}{\sqrt{n}} \\
& \rightarrow_{d} \int_{0}^{T} p_{j}(s) w(s) \alpha_{0}(s)^{-1} y(s)^{-1} d V(s) \sim N\left\{0, \tau(j)^{2}\right\}
\end{aligned}
$$

in which $\tau(j)^{2}=\int_{0}^{T} p_{j}(s)^{2} w(s)^{2} \alpha_{0}(s)^{-2} y(s)^{-1} \alpha(s) d s$. Hence

$$
H(m)=E D(m) \doteq \sum_{j=0}^{m}\left\{c(j)^{2}-\tau(j)^{2} / n\right\}
$$

Furthermore $E \widehat{c}(j)^{2} \doteq c(j)^{2}+\tau(j)^{2} / n$ by the unbiasedness propery, and $\widehat{\tau}(j)^{2}=$ $\int_{0}^{T} p_{j}(s)^{2} w(s)^{2} \alpha_{0}(s)^{-2} \widehat{y}_{n}(s)^{-1} d \widehat{A}_{n}(s)$ estimates $\tau(j)^{2}$, with bias at most $O(1 / n)$. Accordingly

$$
\widehat{H}(m)=\sum_{j=0}^{m}\left\{\widehat{c}(j)^{2}-\frac{2}{n} \widehat{\tau}(j)^{2}\right\}=\sum_{j=0}^{m} \frac{\widehat{\tau}(j)^{2}}{n}\left\{\frac{n \widehat{c}(j)^{2}}{\widehat{\tau}(j)^{2}}-2\right\}
$$


is an approximately unbiased estimate of $H(m)$. The strategy is to compute $\widehat{H}(m)$ for $m=0,1,2, \ldots$ and then choose $m$ to maximise.

It is advisable to use robustified estimators for the $c(j)$ coefficients in cases where $\alpha_{0}(s)$ is small over some of $[0, T]$. Observe that the probability of incorrectly including the $c(j) p_{j}(s)$ term with the (4.5) criterion is close to $\operatorname{Pr}\left\{N(0,1)^{2}>2\right\}=0.157$. Other versions could be more stingy, for example by subtracting $(2+k j) \widehat{\tau}(j)^{2} / n$ instead of $2 \widehat{\tau}(j)^{2} / n$ in (4.5), for suitable constant $k$. This corresponds to using an extra term in $\operatorname{ISE}(m)$ that penalises for including too many terms.

\subsection{NONPARAMETRIC CORRECTION ON A PARAMETRIC START ESTIMATE}

The expansion method can be expected to be best in cases where the initial $\alpha_{0}$ is already close to the truth. Then the correction factor is nearly constant and needs few terms. A natural idea is to employ a parametrically estimated initial step. This can also alleviate problems that the simpler method of 4.1 might run into in cases with bimodal or other difficult shapes for the true hazard. So let $\alpha(s, \theta)$ be a suitable parametric family of hazard rates, as in Section 2. Parts of the theory of 4.1 go through with minor modifications, including construction of the hazard rate estimator itself, but the estimation noise inherent in using $\alpha_{0}(s)=\alpha\left(s, \widehat{\theta}_{n}\right)$ plays a rôle when devising a criterion for choosing the order $m$, and a more sophisticated stopping rule than $(4.5)$ is needed.

Write $\alpha(s)=\alpha\left(s, \widehat{\theta}_{n}\right) \beta(s)$, so that the correction factor this time depends on the parameter estimate. It can nevertheless be expanded in terms of the $p_{j}$ functions,

$$
\beta(s)=\sum_{j=0}^{\infty} c\left(j, \widehat{\theta}_{n}\right) p_{j}(s) \quad \text { with } \quad c\left(j, \widehat{\theta}_{n}\right)=\int_{0}^{T} p_{j}(s) w(s) \alpha\left(s, \widehat{\theta}_{n}\right)^{-1} \alpha(s) d s
$$

where these random coefficients estimate underlying values $c\left(j, \theta_{0}\right)$, featuring the particular parameter value that is least false according to the distance measure (2.4). The natural estimates are

$$
\widehat{c}\left(j, \widehat{\theta}_{n}\right)=\int_{0}^{T} p_{j}(s) w(s) \alpha\left(s, \widehat{\theta}_{n}\right)^{-1} d \widehat{A}_{n}(s)
$$

leading to the semiparametric hazard rate estimator

$$
\alpha_{m}^{*}(s)=\alpha\left(s, \widehat{\theta}_{n}\right) \sum_{j=0}^{m} \widehat{c}\left(j, \widehat{\theta}_{n}\right) p_{j}(s) .
$$

It is not difficult to derive an expression for the integrated squared error for this estimator, in analogy with the reasoning that led to (4.3):

$$
\operatorname{ISE}^{*}(m)=\int_{0}^{T}\left\{\alpha_{m}^{*}(s)-\alpha(s)\right\}^{2} \frac{w(s) d s}{\alpha\left(s, \widehat{\theta}_{n}\right)^{2}}=\sum_{j=0}^{\infty} c\left(j, \widehat{\theta}_{n}\right)^{2}-D^{*}(m)
$$


in which

$$
D^{*}(m)=\sum_{j=0}^{m}\left[c\left(j, \widehat{\theta}_{n}\right)^{2}-\left\{\widehat{c}\left(j, \widehat{\theta}_{n}\right)-c\left(j, \widehat{\theta}_{n}\right)\right\}^{2}\right] .
$$

Again it is natural to choose $m$ to maximise $D^{*}(m)$ or its expected value. But the remaining task is more difficult than in the previous subsection: We need to find a suitable expression $H^{*}(m)$ for the mean value of $D^{*}(m)$ and then an approximately unbiased estimator $\widehat{H}^{*}(m)$ for $H^{*}(m)$.

To find approximate expected values of the terms in $D^{*}(m)$, start out using Taylor expansion to get

$$
\alpha\left(s, \widehat{\theta}_{n}\right)^{-1} \doteq \alpha\left(s, \theta_{0}\right)^{-1}\left\{1-\psi\left(s, \theta_{0}\right)^{\prime}\left(\widehat{\theta}_{n}-\theta_{0}\right)\right\}
$$

This leads to

$$
c\left(j, \widehat{\theta}_{n}\right) \doteq c\left(j, \theta_{0}\right)-b\left(j, \theta_{0}\right)^{\prime}\left(\widehat{\theta}_{n}-\theta_{0}\right),
$$

where $b\left(j, \theta_{0}\right)=\int_{0}^{T} p_{j}(s) w(s) \alpha\left(s, \theta_{0}\right)^{-1} \psi\left(s, \theta_{0}\right) \alpha(s) d s$, from which it follows that

$$
\sqrt{n}\left\{c\left(j, \widehat{\theta}_{n}\right)-c\left(j, \theta_{0}\right)\right\} \rightarrow_{d} N\left\{0, b\left(j, \theta_{0}\right)^{\prime} J^{-1} K J^{-1} b\left(j, \theta_{0}\right)\right\}
$$

upon using (2.5) from Section 2 .

Let us pause briefly to make a useful general point about de-biasing and expected squares. Suppose $T_{n}$ estimates the parameter $a$ and has $E T_{n} \doteq a+b / n$ and $\sqrt{n}\left(T_{n}-a\right) \rightarrow_{d} N\left\{0, \tau^{2}\right\}$. An approximation is then $E T_{n}^{2} \doteq a^{2}+\tau^{2} / n+2 a b / n$. But after de-biasing $T_{n}$ according to some scheme to get $\left\langle T_{n}\right\rangle_{\mathrm{db}}=T_{n}-\widehat{b} / n$, say, then typically

$$
\sqrt{n}\left(\left\langle T_{n}\right\rangle_{\mathrm{db}}-a\right) \rightarrow_{d} N\left\{0, \tau^{2}\right\} \quad \text { and } \quad E\left\langle T_{n}\right\rangle_{\mathrm{db}}^{2} \doteq a^{2}+\tau^{2} / n
$$

[where $\left\langle T_{n}\right\rangle_{\mathrm{db}}^{2}$ means $\left\{\left\langle T_{n}\right\rangle_{\mathrm{db}}\right\}^{2}$ ]. Several de-biasing schemes succeed in achieving this, for example the jackknife and the bootstrap method. This way an explicit expression for $E\left\langle T_{n}\right\rangle_{\mathrm{db}}^{2}$ is obtained without knowing or caring about the actual value of $b$.

By these arguments and (4.8) we see that after de-biasing of $\widehat{\theta}_{n}, E c\left(j,\left\langle\widehat{\theta}_{n}\right\rangle_{\mathrm{db}}\right)^{2} \doteq$ $c\left(j, \theta_{0}\right)^{2}+b\left(j, \theta_{0}\right)^{\prime} J^{-1} K J^{-1} b\left(j, \theta_{0}\right) / n$. Next another Taylor approximation, with $B_{n}(j)=\int_{0}^{T} p_{j}(s) w(s) \alpha\left(s, \theta_{0}\right)^{-1} \psi\left(s, \theta_{0}\right)^{\prime} d \widehat{A}_{n}(s)$, leads to

$$
\begin{aligned}
\widehat{c}\left(j, \widehat{\theta}_{n}\right)-c\left(j, \widehat{\theta}_{n}\right) & \doteq \widehat{c}\left(j, \theta_{0}\right)-B_{n}(j)^{\prime}\left(\widehat{\theta}_{n}-\theta_{0}\right)-c\left(j, \theta_{0}\right)+b\left(j, \theta_{0}\right)^{\prime}\left(\widehat{\theta}_{n}-\theta_{0}\right) \\
& =\int_{0}^{T} p_{j}(s) w(s) \frac{d \widehat{A}_{n}(s)-d A(s)}{\alpha\left(s, \theta_{0}\right)}-\left\{B_{n}(j)-b\left(j, \theta_{0}\right)\right\}^{\prime}\left(\widehat{\theta}_{n}-\theta_{0}\right),
\end{aligned}
$$


which is seen to imply

$$
\sqrt{n}\left\{\widehat{c}\left(j, \widehat{\theta}_{n}\right)-c\left(j, \widehat{\theta}_{n}\right)\right\} \rightarrow_{d} \int_{0}^{T} p_{j}(s) w(s) \alpha\left(s, \theta_{0}\right)^{-1} y(s)^{-1} d V(s) .
$$

This variable has variance $\tau\left(j, \theta_{0}\right)^{2}=\int_{0}^{T} p_{j}(s)^{2} w(s)^{2} \alpha\left(s, \theta_{0}\right)^{-2} y(s)^{-1} d A(s)$. We may sum this part up by writing

$$
H^{*}(m)=E D^{*}(m) \doteq \sum_{j=0}^{m}\left\{c\left(j, \theta_{0}\right)^{2}-\frac{1}{n} \tau\left(j, \theta_{0}\right)^{2}+\frac{1}{n} b\left(j, \theta_{0}\right)^{\prime} J^{-1} K J^{-1} b\left(j, \theta_{0}\right)\right\}
$$

Compare (4.4).

It remains to find an approximately unbiased estimator for the terms of this sum. It is natural to use $\widehat{c}\left(j, \widehat{\theta}_{n}\right)$ and its square. From (2.2) with Taylor comes

$$
\begin{aligned}
\widehat{c}\left(j, \widehat{\theta}_{n}\right) & \doteq \int_{0}^{T} p_{j}(s) \frac{w(s)}{\alpha\left(s, \theta_{0}\right)}\left\{1-\psi\left(s, \theta_{0}\right)^{\prime}\left(\widehat{\theta}_{n}-\theta_{0}\right)\right\}\left\{d A(s)+d \widehat{A}_{n}(s)-d A(s)\right\} \\
& \doteq{ }_{d} c\left(j, \theta_{0}\right)+\int_{0}^{T} p_{j}(s) \frac{w(s)}{\alpha\left(s, \theta_{0}\right)} \frac{n}{Y_{n}(s)} \frac{d M_{n}(s)}{\sqrt{n}}-b\left(j, \theta_{0}\right)^{\prime}\left(\widehat{\theta}_{n}-\theta_{0}\right),
\end{aligned}
$$

and yields, in conjunction with (2.5), that

$$
\begin{aligned}
\sqrt{n}\left\{\widehat{c}\left(j, \widehat{\theta}_{n}\right)\right. & \left.-c\left(j, \theta_{0}\right)\right\} \rightarrow d \int_{0}^{T} p_{j}(s) w(s) \alpha\left(s, \theta_{0}\right)^{-1} y(s)^{-1} d V(s) \\
& -b\left(j, \theta_{0}\right)^{\prime} J^{-1} \int_{0}^{T} \psi\left(s, \theta_{0}\right)\left[d V(s)+Z(s)\left\{\alpha(s)-\alpha\left(s, \theta_{0}\right)\right\} d s\right] .
\end{aligned}
$$

The variance of this limit variable can be shown to be

$$
\tau\left(j, \theta_{0}\right)^{2}+b\left(j, \theta_{0}\right)^{\prime} J^{-1} K J^{-1} b\left(j, \theta_{0}\right)-2 b\left(j, \theta_{0}\right)^{\prime} J^{-1} b\left(j, \theta_{0}\right)-2 b\left(j, \theta_{0}\right)^{\prime} J^{-1} d\left(j, \theta_{0}\right),
$$

in which $d\left(j, \theta_{0}\right)=\int_{0}^{T} p_{j}(s) w(s)\left\{\alpha(s) / \alpha\left(s, \theta_{0}\right)\right\} y(s)^{-1} E(s) d A(s)$, again using the $E(s)$ function defined after (2.4). These efforts lead to

$$
\begin{array}{r}
E\left\langle\widehat{c}\left(j, \widehat{\theta}_{n}\right)\right\rangle_{\mathrm{db}}^{2} \doteq c\left(j, \theta_{0}\right)^{2}+\left\{\tau\left(j, \theta_{0}\right)^{2}+b\left(j, \theta_{0}\right)^{\prime} J^{-1} K J^{-1} b\left(j, \theta_{0}\right)\right. \\
\left.-2 b\left(j, \theta_{0}\right)^{\prime} J^{-1} b\left(j, \theta_{0}\right)-2 b\left(j, \theta_{0}\right)^{\prime} J^{-1} d\left(j, \theta_{0}\right)\right\} / n
\end{array}
$$

by the de-biasing trick (4.9). And in the end this invites

$$
\begin{aligned}
\widehat{H}^{*}(m)=\sum_{j=0}^{m}\left\{\left\langle\widehat{c}\left(j, \widehat{\theta}_{n}\right)\right\rangle_{\mathrm{db}}^{2}-\frac{2}{n} \widehat{\tau}\left(j, \widehat{\theta}_{n}\right)^{2}\right. \\
\left.+\frac{2}{n} \widehat{b}\left(j, \widehat{\theta}_{n}\right)^{\prime} \widehat{J}_{n}^{-1} \widehat{b}\left(j, \widehat{\theta}_{n}\right)+\frac{2}{n} \widehat{d}\left(j, \widehat{\theta}_{n}\right)^{\prime} \widehat{J}_{n}^{-1} \widehat{b}\left(j, \widehat{\theta}_{n}\right)\right\}
\end{aligned}
$$


to be used to estimate $H^{*}(m)$ above. Here $\widehat{\tau}\left(j, \widehat{\theta}_{n}\right), \widehat{b}\left(j, \widehat{\theta}_{n}\right), \widehat{d}\left(j, \widehat{\theta}_{n}\right)$, and $\widehat{J}_{n}$ are the natural empirical analogues to $\tau\left(j, \theta_{0}\right), b\left(j, \theta_{0}\right), d\left(j, \theta_{0}\right)$, and $J$, and emerge by replacing $d A(s)$ with $d \widehat{A}_{n}(s)$ and $\theta_{0}$ with $\widehat{\theta}_{n}$.

Some of the comments ending the previous subsection are relevant here too. Note from (4.6) that if $\alpha(s, \theta)=\theta$, then $\widehat{\theta}_{n}$ cancels out, so only models with non-constant hazard rates are of interest. We remark finally that there are other ways of trying to find the $m$ that gives largest $D^{*}(m)$ of (4.7). One such method is to bootstrap. View $D^{*}(m)$ as a complicated function $S_{m}\left(A, \widehat{\theta}_{n}\right)$ depending on both $A$ and the data, via $\widehat{\theta}_{n}$. Obtain 1000 bootstrap versions $S_{m}\left(\widehat{A}_{n}, \widehat{\theta}_{n}^{*}\right)$ and take average, and choose $m$ in the end to maximise. See Hjort (1985b) for some bootstrapping theory for censored data.

\section{Bayesian uncertainty around a parametric hazard rate model}

This section provides two frameworks where a semiparametric prior is put on the space of all distributions, centred at a given parametric hazard rate model. The first version uses a Dirichlet prior on a particular residual distribution, applying ideas of Hjort (1986c, 1987). The second version creates Bayesian uncertainty around a proportional hazard type model, using a Beta process prior (from Hjort, 1990b) on a basis hazard and a parametric prior on the proportionality factors. The prior processes we use will produce non-continuous sample paths, so we prefer using the definition

$$
d A(s)=\frac{F[s, s+d s]}{F[s, \infty)} \text { and } A(t)=\int_{0}^{t} \frac{d F(s)}{F[s, \infty)}
$$

instead of (1.1) for the cumulative hazard function $A$. Its solution for $F$ is $F(t)=$ $1-\prod_{[0, t]}\{1-d A(s)\}$, featuring the product integral, see Hjort (1990b) and Gill and Johansen (1990).

\subsection{DiRICHLET PRIOR FOR THE RESIDUAL DISTRIBUtion}

One way of describing the familiar exponential model for life-times $X_{i}^{0}$ is to say that $X_{i}^{0}=Y_{i} / \theta$, where the $Y_{i}^{\prime}$ 's are i.i.d. from $G_{0}(y)=1-\exp (-y)$. Think about the $Y_{i}$ 's as 'standardised residuals' and their distribution $G$ as the residual distribution. A Bayesian framework for an uncertain exponential model is to place a parametric prior $\pi(\theta) d \theta$ on $\theta$ and a nonparametric prior on $G$, centred at $G=G_{0}$. Thus $X_{i}^{0}$ has cumulative $F(t)=G(\theta t)$, expressed in terms of the two unknown parameters, one finite-dimensional and one infinite-dimensional. Let $G$ be a Dirichlet process with parameter $a G_{0}$, and stipulate that $\theta$ and $G$ are independent. The purely parametric $G_{0}(\theta t)$ corresponds to the limit as $a \rightarrow \infty$.

The following results can be obtained, using methods of Hjort (1986c, 1987). We assume in this subsection that there is no censoring, and that the data points $x_{i}=$ 
$x_{i}^{0}$ are distinct, mainly for clarity of presentation. First, the posterior distribution for $\theta$ alone is $\pi_{n}(\theta)=$ const. $\pi(\theta) L_{n}(\theta)$, where $L_{n}(\theta)=\prod_{i=1}^{n}\left\{g_{0}\left(\theta x_{i}\right) \theta\right\}$. This happens to be identical to the posterior density that would have been obtained without the extra randomness in $G$ around $G_{0}$. The posterior of $G$ is a continuous mixture of Dirichlet processes, since

$$
G \mid\{\text { data, } \theta\} \sim \operatorname{Dirichlet}\left\{a G_{0}+\sum_{i=1}^{n} I\left(\theta x_{i}\right)\right\},
$$

where $I\left(\theta x_{i}\right)$ denotes unit point mass at the point $\theta x_{i}$. Its expected value is

$$
G_{n}^{*}(t)=E\{G(t) \mid \text { data }\}=w_{n} G_{0}(t)+\left(1-w_{n}\right) \frac{1}{n} \sum_{i=1}^{n} \operatorname{Pr}\left\{\theta x_{i} \leq t \mid \text { data }\right\}
$$

where $w_{n}=a /(a+n)$. If $\theta$ has a Gamma prior with parameters $(b, c)$, for example, then $\pi_{n}($.$) is Gamma with \left(b+n, c+n \bar{x}_{n}\right)$, and we see that $G_{n}^{*}($.$) has a density$

$$
g_{n}^{*}(t)=w_{n} g_{0}(t)+\left(1-w_{n}\right) \frac{1}{n} \sum_{i=1}^{n} \gamma\left(t / x_{i} ; b+n, c+n \bar{x}_{n}\right) / x_{i}
$$

in which $\gamma(t ; b, c)$ denotes the gamma density. An approximation for $n$ large is

$$
g_{n}^{*}(t) \doteq w_{n} g_{0}(t)+\left(1-w_{n}\right) \frac{1}{n} \sum_{i=1}^{n} \phi\left(\frac{t-x_{i} \widehat{\theta}_{n}}{h_{i}}\right) \frac{1}{h_{i}}
$$

where $\widehat{\theta}_{n}=1 / \bar{x}_{n}$ is the maximum likelihood estimator and $h_{i}=x_{i} \widehat{\theta}_{n} / \sqrt{n}$. This is a variable kernel density estimate of the residual distribution, using estimated residuals $\widehat{y}_{i}=x_{i} \widehat{\theta}_{n}$ and local bandwidths $h_{i}=\widehat{y}_{i} / \sqrt{n}$.

The natural semiparametric Bayesian estimate of the hazard rate would be the derivative $\alpha_{n}^{*}(s)$ of a smoothed $A_{n}^{*}(t)=E\{A(t) \mid$ data $\}$. There is no explicit formula for this, but simulations can be carried out to give an answer. Draw $\theta$ from $\pi_{n}($.$) ,$ then draw $G$ from (5.2), and use $F(t)=G(\theta t)$ with (5.1) to produce one particular $A$. Do this some thousand times and compute the average, and smooth and differentiate in the end. - An easier way out is to calculate the exact $F_{n}^{*}(t)=E\{F(t) \mid$ data $\}$ instead, which is possible, and then use (5.1) or a smoothed version to give $\alpha_{n}^{*}(s)$. One finds $F_{n}^{*}(t)=w_{n} E\left\{G_{0}(t \theta) \mid\right.$ data $\}+\left(1-w_{n}\right) F_{n}(t)$, in which $F_{n}$ is the empirical distribution. This leads to

$$
d A_{n}^{*}(s) \doteq \frac{w_{n} \exp \left(-\widehat{\theta}_{n} s\right) \widehat{\theta}_{n} d s+\left(1-w_{n}\right) d N_{n}(s) / n}{w_{n} \exp \left(-\widehat{\theta}_{n} s\right)+\left(1-w_{n}\right) Y_{n}(s) / n}
$$

which upon smoothing gives the formula

$$
\alpha_{n}^{*}(s)=k_{n}(s) \hat{\theta}_{n}+\left\{1-k_{n}(s)\right\} \tilde{\alpha}_{n}(s) .
$$


Here $\tilde{\alpha}_{n}(s)$ is a smoothed Nelson-Aalen, like the Ramlau-Hansen version described in (3.13), and the weight factor favouring the parametric estimate is $k_{n}(s)=$ $w_{n} \exp \left(-\widehat{\theta}_{n} s\right) /\left\{w_{n} \exp \left(-\widehat{\theta}_{n} s\right)+\left(1-w_{n}\right) Y_{n}(s) / n\right\}$

It remains here to specify the choice of $w_{n}=a /(a+n)$. The ideal Bayesian determines $a$ from 'prior considerations'; it has interpretation as 'prior sample size' or strength of belief in the Dirichlet prior. The $a$ parameter could be determined from a measure of how well the data fit the parametric $\alpha(s, \theta)=\theta$; if data support the model then $a$ should be large and vice versa. General goodness of fit measures are in Hjort (1990a). It can also be determined via empirical Bayesian ideas, as briefly discussed in Hjort (1991b). A double bootstrap method for a rather similar problem was suggested in Hjort (1988).

The scheme above works for other parametric families as well, where the starting point is to regard $X_{i}^{0}$ as $A^{-1}\left(Y_{i}, \theta\right)$, where the residuals $Y_{i}$ come from $G$, which has a Dirichlet prior centred at $G=G_{0}$. Here $A^{-1}(y, \theta)$ is the inverse of $\int_{0}^{x} \alpha(s, \theta) d s$. Again the posterior $\pi_{n}(\theta)$ is as under the null model $G=G_{0}$, and the Bayes estimate $G_{n}^{*}(t)$ has a density which is a mixture of $g_{0}(t)=\exp (-t)$ and a function which is close to being a variable kernel density estimate of the estimated residuals $\widehat{y}_{i}=$ $A\left(x_{i}^{0}, \widehat{\theta}_{n}\right)$, with local bandwidths proportional to $1 / \sqrt{n}$. And the Bayes estimate $F_{n}^{*}$ of $F$ is the pleasing mixture $w_{n} \int F_{0}(., \theta) \pi_{n}(\theta) d \theta+\left(1-w_{n}\right) F_{n}$, where $F_{0}(t, \theta)=$ $G_{0}(A(t, \theta))$ is the null model for $F$. [This, incidentally, can be viewed as providing a semiparametric Bayesian justification for an ad hoc method suggested in Olkin and Spiegelman (1987) and Hjort (1988).] Finally the semiparametric hazard rate estimate generalising $(5.4)$ is

$$
\begin{aligned}
\alpha_{n}^{*}(s)= & \frac{w_{n} \exp \left\{-A\left(s, \widehat{\theta}_{n}\right)\right\}}{w_{n} \exp \left\{-A\left(s, \widehat{\theta}_{n}\right)\right\}+\left(1-w_{n}\right) Y_{n}(s) / n} \alpha\left(s, \widehat{\theta}_{n}\right) \\
& +\frac{\left(1-w_{n}\right) Y_{n}(s) / n}{w_{n} \exp \left\{-A\left(s, \widehat{\theta}_{n}\right)\right\}+\left(1-w_{n}\right) Y_{n}(s) / n} \tilde{\alpha}_{n}(s) .
\end{aligned}
$$

The regime can also be extended to handle the Cox regression setup where under the null model $\alpha_{i}(s)=\alpha(s, \theta) \exp \left(\beta^{\prime} z_{i}\right)$ for individual no. $i$. Some technical details are necessary to go through here; see Hjort (1991b, Section 7 ) for similar results and techniques carried out for ordinary linear regression. We mention finally that fuller Bayesian analysis (as opposed to only getting an estimate) would be possible using Gibbs sampling techniques to sample from the posterior distribution of the parameter of interest.

\subsection{BETA PROCESS PRIOR FOR THE BASIC HAZARD}

Suppose individual no. $i$ has life-time $X_{i}^{0}$ with cumulative hazard $A_{i}($.$) , interpreted$ as in (5.1). Let us postulate that

$$
1-d A_{i}(s)=\{1-d A(s)\}^{g_{i}(s, \theta)},
$$


for suitable 'relative risk factor function' $g_{i}(s, \theta)$, where $A($.$) is a random cumulative$ hazard process centred at some $A_{0}=\int_{0}^{\cdot} \alpha_{0}(s) d s$. Suppose a new individual has relative risk factor $g(s, \theta)$. This individual has survival function

$$
F([t, \infty) \mid g)=\prod_{[0, t)}\{1-d A(s \mid g)\}=\prod_{[0, t)}\{1-d A(s)\}^{g(s, \theta)}
$$

Among our present aims is to develop a semiparametric estimate of $\alpha(s \mid g)$, the hazard rate for this $g$-individual, by working in a suitable nonparametric model neighbourhood around the null model that corresponds to $A=A_{0}$. Under the null model $\alpha_{i}(s)=\alpha_{0}(s) g_{i}(s, \theta)$ for no. $i$ and $\alpha(s \mid g)=\alpha_{0}(s) g(s, \theta)$ for the $g$-person.

The $g_{i}$ factor would typically be modelled as a function of some covariate vector $z_{i}$ associated with individual $i$. A prime example of the setup above is the proportional hazard type $g_{i}(s)=\alpha(s, \gamma) \exp \left(\beta^{\prime} z_{i}\right)$ (where $\gamma$ and $\beta$ together make up $\theta$ ) and where $A_{0}(t)=t$. The semiparametric Bayesian model amounts in this case to a random perturbation of the parametric Cox model under which the $z$-person has hazard rate $\alpha(s \mid z)=\alpha(s, \gamma) \exp \left(\beta^{\prime} z\right)$.

The most natural class of priors to choose from is that of the Beta processes, see Hjort (1990b). Let $A($.$) be a Beta process with parameters c($.$) and A_{0}($.$) , which$ means that $A($.$) has independent increments that are nearly Beta distributed,$

$$
d A(s) \doteq_{d} \operatorname{Beta}\left\{c(s) d A_{0}(s), c(s)\left(1-d A_{0}(s)\right)\right\} .
$$

Note that $E d A(s)=d A_{0}(s)$ and that $\operatorname{Var} d A(s)=d A_{0}(s) /\{1+c(s)\}$, i.e. $A($.$) is$ a random cumulative hazard process that we may expect to be in the vicinity of $A_{0}($.$) . If the values of c$ are large then $A(.) \doteq A_{0}($.$) and the null model prevails.$

As in 5.1 we can let $\theta$ have prior $\pi(\theta)$, independently of the Beta process for $A$, and with some efforts calculate the posterior distribution of $A$ given data and $\theta$, and then the posterior distribution of $\theta$ alone. This makes it possible to compute a Bayes estimate of the hazard rate, and of other quantities of interest. In this subsection censoring is allowed, as in the paragraph containing (1.1).

Introduce $R_{n}(s, \theta)=\sum_{i=1}^{n} Y_{i}(s) g_{i}(s, \theta)$, the sum of relative hazards for those under risk, using $Y_{i}(s)=I\left\{X_{i}^{0} \geq s, C_{i} \geq s\right\}$ as at-risk indicator for no. $i$. Let also $R_{n, i}(s, \theta)=\sum_{j \neq i} Y_{j}(s) g_{j}(s, \theta)$ be the corresponding sum where no. $i$ is excluded. The data set is of the form $X_{i}>x_{i}$ for those with $\delta_{i}=0$ and $X_{i}=x_{i}$ for those with $\delta_{i}=1$. Calculation of the posterior distribution of $(A, \theta)$ is simplest when conditioning on the extended event $X_{i}>x_{i}$ for those with $\delta_{i}=0$ and $X_{i} \in\left[x_{i}, x_{i}+\varepsilon\right]$ for those with $\delta_{i}=1$, and then letting $\varepsilon \rightarrow 0$ in the end. This event has positive 
probability

$$
\begin{aligned}
L(\text { data } \mid \theta, A) & =\prod_{\text {outside }}\{1-d A(s)\}^{R_{n}(s, \theta)} \\
\times & \prod_{i: \delta_{i}=1}\left[\prod_{\left[x_{i}, x_{i}+\varepsilon\right]}\{1-d A(s)\}^{R_{n, i}(s, \theta)}-\prod_{\left[x_{i}, x_{i}+\varepsilon\right]}\{1-d A(s)\}^{R_{n}(s, \theta)}\right]
\end{aligned}
$$

for given $\theta$ and $A$, where the 'outside' set is the observation interval $[0, T]$ minus the small $\left[x_{i}, x_{i}+\varepsilon\right]$ intervals. Techniques from Hjort (1990b) can be used to prove that $A$ given data and $\theta$ still is Lévy, i.e. has independent increments, with distributions given by

$$
d A(s) \mid\{\text { data, } \theta\} \doteq_{d} \operatorname{Beta}\left\{c(s) d A_{0}(s), c(s)\left(1-d A_{0}(s)\right)+R_{n}(s, \theta)\right\}
$$

for $s$ outside observed life-times $x_{i}$, while the jump $\Delta A\left(x_{i}\right)=A\left\{x_{i}\right\}$ at an observed life-time is distributed with density

$$
f(z)=\frac{z^{-1}(1-z)^{c\left(x_{i}\right)+R_{n, i}\left(x_{i}, \theta\right)-1}\left\{1-(1-z)^{g_{i}\left(x_{i}, \theta\right)}\right\}}{\psi\left(c\left(x_{i}\right)+R_{n}\left(x_{i}, \theta\right)\right)-\psi\left(c\left(x_{i}\right)+R_{n, i}\left(x_{i}, \theta\right)\right)}, \quad 0<z<1 .
$$

Here $\psi(u)=\Gamma^{\prime}(u) / \Gamma(u)$ is the digamma function, and it is assumed that the $x_{i}$ 's are distinct. The expected value of this jump can be calculated to be $J_{n}\left(x_{i}, \theta\right) /\left\{c\left(x_{i}\right)+\right.$ $\left.R_{n}\left(x_{i}, \theta\right)\right\}$, where

$$
J_{n}\left(x_{i}, \theta\right)=\frac{g_{i}\left(x_{i}, \theta\right)}{c\left(x_{i}\right)+R_{n, i}\left(x_{i}, \theta\right)}\left\{\psi\left(c\left(x_{i}\right)+R_{n}\left(x_{i}, \theta\right)\right)-\psi\left(c\left(x_{i}\right)+R_{n, i}\left(x_{i}, \theta\right)\right)\right\}^{-1}
$$

so that

$$
E\{A(t) \mid \text { data, } \theta\}=\int_{0}^{t} \frac{c(s) d A_{0}(s)+J_{n}(s, \theta) d N_{n}(s)}{c(s)+R_{n}(s, \theta)}
$$

(where we may take $J_{n}(s, \theta)=0$ outside the observed life-times). Since $\psi(x) \doteq$ $\log \left(x+\frac{1}{6}\right)$ for $x$ not too small and $R_{n}\left(x_{i}, \theta\right)=R_{n, i}\left(x_{i}, \theta\right)+g_{i}\left(x_{i}, \theta\right)$ one sees that $J_{n}\left(x_{i}, \theta\right)$ is roughly close to 1 .

We can also obtain the expected value of the $g$-survival function $p=F([t, \infty) \mid g)$, given data and $\theta$, from (5.7) and the results above. After carefully tending to both factors in

$$
E\{p \mid \text { data, } \theta\}=E\left[\prod_{\text {outside }}\{1-d A(s)\}^{g(s, \theta)} \prod_{i: \delta_{i}=1, x_{i}<t}\left\{1-\Delta A\left(x_{i}\right)\right\}^{g\left(x_{i}, \theta\right)}\right],
$$


where the first product is over $[0, t)$ minus the data points, one finds

$$
\begin{aligned}
& \exp \left[-\int_{0}^{t}\left\{\psi\left(c(s)+R_{n}(s, \theta)+g(s, \theta)\right)-\psi\left(c(s)+R_{n}(s, \theta)\right)\right\} c(s) d A_{0}(s)\right] \\
& \times \prod_{i: x_{i}<t}\left[\frac{\psi\left(c\left(x_{i}\right)+R_{n}\left(x_{i}, \theta\right)+g\left(x_{i}, \theta\right)\right)-\psi\left(c\left(x_{i}\right)+R_{n, i}\left(x_{i}, \theta\right)+g\left(x_{i}, \theta\right)\right)}{\psi\left(c\left(x_{i}\right)+R_{n}\left(x_{i}, \theta\right)\right)-\psi\left(c\left(x_{i}\right)+R_{n, i}\left(x_{i}, \theta\right)\right)}\right]^{\delta_{i}} .
\end{aligned}
$$

If $n$ is large and/or $c($.$) is small the first term becomes 1$ while a somewhat crude approximation to the second term is the Kaplan-Meier-type $\prod_{[0, t)}\left[1-g(s, \theta) d N_{n}(s) /\right.$ $\left.\left\{R_{n}(s, \theta)+g(s, \theta)\right\}\right]$.

To solve the semiparametric Bayesian problem it remains to find the posterior distribution of $\theta$ alone. The answer can be shown to be

$$
\begin{aligned}
\pi_{n}(\theta) & =\text { const. } \pi(\theta) \exp \left[-\int_{0}^{T}\left\{\psi\left(c(s)+R_{n}(s, \theta)\right)-\psi(c(s))\right\} c(s) d A_{0}(s)\right] \\
& \times \prod_{i=1}^{n}\left[\left\{\psi\left(c\left(x_{i}\right)+R_{n}\left(x_{i}, \theta\right)\right)-\psi\left(c\left(x_{i}\right)+R_{n, i}\left(x_{i}, \theta\right)\right)\right\} c\left(x_{i}\right) \alpha_{0}\left(x_{i}\right)\right]^{\delta_{i}}
\end{aligned}
$$

see again Hjort (1990b, Section 6) for some necessary techniques. Of course the $c\left(x_{i}\right) a_{0}\left(x_{i}\right)$ terms can be subsumed into the const. here, but they are left in to suggest the necessary changes to come in 5.3 below. A natural semiparametric Bayes solution $p_{n}^{*}$ for $p=F([t, \infty) \mid g)$ for this $g$-individual now emerges by integrating (5.12) w.r.t. this posterior density. In the end one should use smoothing of the analogue of (5.1) to produce the semiparametric Bayes estimator $\alpha_{n}^{*}(s \mid g)$. Fuller Bayesian analysis can be accomplished via a Gibbs sampling regime from the posterior distribution of $(A(),. \theta)$.

Again one must specify the $c($.$) function of the prior process. It has a strength$ of belief Bayesian interpretation and could be specified subjectively as such. One might also model it as $c(s)=c$ or perhaps as $c(s)=a \exp (-b s)$ and reach values for these parameters via empirical Bayes methods. An attractive general possibility is to use a vague prior for $\theta$ in conjunction with letting $c(.) \rightarrow 0$, treating the resulting methods as Bayesian solutions under a noninformative reference prior. See Hjort (1986a, Section 4) for some Jeffreys prior calculations.

Note that $\pi_{n}(\theta)$ becomes proportional to $\exp \left\{-\int_{0}^{T} R_{n}(s, \theta) d A_{0}(s)\right\} \prod_{i=1}^{n} g_{i}\left(x_{i}\right.$, $\theta)^{\delta_{i}}$, which is the ordinary likelihood under the null model, in the limit as $c(.) \rightarrow \infty$. In cases where $c($.$) is small and/or n$ large the first factor of (5.13) becomes less and less informative for $\theta$ while a first order approximation to the second factor is the familiar partial likelihood $\prod_{i: \delta_{i}=1}\left\{g_{i}\left(x_{i}, \theta\right) / R_{n}\left(x_{i}, \theta\right)\right\}$. In the special case of $g_{i}(s)=\alpha(s, \gamma) \exp \left(\beta^{\prime} z_{i}\right)$ we see that the common $\alpha(s, \gamma)$ factors out, and the data 
do not give extra information on this term, only for the $\beta$ coefficients. The $\beta$ part, relating to the inhomogeneity aspects of the model, works well, in that a Bayesian will reach the same conclusions as would a frequentist when more and more data come in. This can be proved using methods of Hjort (1986a, Section 4; 1990b, Section 6). In the homogeneous case, however, with $g_{i}(s, \theta)=\theta$, we have another example where the Bayesian machinery is inconsistent in an infinite-dimensional problem.

\subsection{BETA PROCESS PRIOR DEPENDING ON THE PARAMETER}

The semiparametric Bayesian setup of 5.2 worked well regarding analysis of those aspects of the model that correspond to inhomogeneity, but not in the fully homogeneous model, for example. Another version with somewhat better properties regarding the homogeneity aspects is as follows. Let again $\theta$ have prior $\pi(\theta)$, but let $A$ given $\theta$ be a Beta process with parameters $c($.$) and A(., \theta)=\int_{0}^{\cdot} \alpha(s, \theta) d s$, i.e., centred at the hazard rate $\alpha(s, \theta)$ for given $\theta$. Let otherwise the situation be as in the previous subsection. This framework amounts to having a nonparametric prior around the parametric $\alpha(s \mid g)=\alpha(s, \theta) g(s, \theta)$, in contrast to $\alpha(s \mid g)=\alpha_{0}(s) g(s, \theta)$ for 5.2.

The necessary details resemble those of the previous subsection, and we skip them here. Formulae (5.8)-(5.13) typically become valid in the present setting when $d A_{0}(s)$ is replaced with $\alpha(s, \theta) d s$ and when $\alpha_{0}\left(x_{i}\right)$ is replaced with $\alpha\left(x_{i}, \theta\right)$. As before Bayes estimates can be produced for $A$ and for $F([t, \infty) \mid g)$ and for its accompanying $g$-hazard rate, and a full Bayesian analysis of any quantity of interest is possible through Gibbs sampling from the posterior of $(A(),. \theta)$.

The present setup is somewhat more flexible than that of 5.2 , and is better but really not fully equipped to handle the homogeneous parameters of a Cox-structured $\alpha_{i}(s, \gamma, \beta)=\alpha(s, \gamma) \exp \left(\beta^{\prime} z_{i}\right)$, for example. The Bayes estimators for $\gamma$ can easily be plainly inconsistent. This is easiest to see in the fully homogeneous model where $\alpha(s, \theta)=\theta$ and each $g_{i}(s, \theta)=1$. In this case the Bayes estimate of $\theta$ tends to infinity with increasing $n$, under mild assumptions.

Alternative constructions are necessary in order to get fully robust and consistent priors. One might try other cumulative hazard prior processes, from the Lévy processes discussed in Hjort (1990b), or try out 'pinning down the prior' as in Hjort (1986c). These matters will be pursued in future work.

\section{Additional remarks}

6A. If a parametric model fits the data nicely then a parametric method suffices. Several classes of goodness of fit tests were developed and discussed in Hjort (1990a). $6 \mathrm{~B}$. Such a parametric model does not have to be fully perfect in order for the methods based on it to be better than more conservative ones. In Hjort (1991c) a 
'tolerance distance' is calculated from a moderately incorrect model to a wider and correct one; inside the tolerance radius estimators based on the incorrect model are better than those based on the correct model. For an example, suppose the true model is the gamma one, with hazard function inherited from the density $f(s, \theta, \gamma)=\left\{\theta^{\gamma} / \Gamma(\gamma)\right\} s^{\gamma-1} \exp (-\theta s)$. Then estimators based on the incorrect assumption of a constant rate (which corresponds to $\gamma=1$ ) are better than the two-parameter methods if $|\gamma-1| \leq 1.245 / \sqrt{n}$ (assuming no censoring). This can be seen as still another argument for not giving up simple parametric methods, even though the underlying models might be wrong.

6C. Methods and results of this paper can be generalised in various directions. They could be developed for Aalen's general multiplicative intensity model for counting processes, see Andersen and Borgan (1985) and Andersen et al. (1991) for thorough accounts. In another direction our results could be extended to the full halfline $[0, \infty)$ with appropriate extra assumptions on the censoring mechanism. For the orthogonal expansion methods one might use the Laguerre polynomials which are orthogonal w.r.t. the exponential density. Finally results can be extended from the i.i.d. framework to regression models of the Cox variety. Orthogonal expansion estimators for the unknown basis hazard function in Cox' regression model are for instance easy to construct.

6D. The local likelihood methods of Section 3 apply to density estimation as well. Methods given there can be used to obtain a locally estimated normal density of the type $f(t)=N\left\{\widehat{\mu}_{n}(t), \widehat{\sigma}_{n}(t)^{2}\right\}(t)$, for example.

$6 \mathrm{E}$. Good ideas can sometimes be iterated. For the method of 4.2 this would mean constructing estimators of the type $\widehat{\alpha}(s)=\alpha\left(s, \widehat{\theta}_{n}\right) \widehat{\beta}_{1}(s) \cdots \widehat{\beta}_{k}(s)$, where each successive correction factor perhaps should use only a small number of terms. This resembles projection pursuit. Good stopping rules could be based on cross validation, for example, but would be somewhat cumbersome.

$6 \mathrm{~F}$. A simulation study comparing the various estimators would be welcome. Some of the questions to answer include: Are the new estimators better than the nonparametric ones when the true hazard is close to the parametric model used? How much do they lose to the parametric ones on the latter's home turf? Are there advantages to using multi-parameter models for the dynamic likelihood methods of Section 3? What are the most useful ways of choosing bandwidth $h_{n}(s)$ for the likelihood methods and weight factor $w_{n}$ in the mixture type estimates (5.5)?

\section{References}

Abramowitz, M. and Stegun, I.A. (1964). Handbook of Mathematical Functions. National Bureau of Standards, Washington.

Andersen, P.K. and Borgan, $\emptyset$. (1985). Counting process models for life history data: A 
review (with discussion). Scand. J. Statist. 12, 97-158.

Andersen, P.K., Borgan, Ø., Gill, R.D., and Keiding, N.L. (1991). Statistical Models Based on Counting Processes. Springer Verlag, to appear.

Borgan, $\emptyset$. (1984). Maximum likelihood estimation in parametric counting process models, with applications to censored failure time data. Scand. J. Statist. 11, 1-16. Corrigendum, ibid. p. 275.

Gill, R.D. and Johansen, S. (1990). A survey of product-integration with a view toward application in survival analysis. Ann. Statist. 18, 1501-1555.

Hjort, N.L. (1985a). Contribution to the discussion of Andersen and Borgan's 'Counting process models for life history data: a review'. Scand. J. Statist. 12, 141-150.

Hjort, N.L. (1985b). Bootstrapping Cox's regression model. Technical Report NSF-241, Department of Statistics, Stanford University.

Hjort, N.L. (1986a). Bayes estimators and asymptotic efficiency in parametric counting process models. Scand. J. Statist. 13, 63-85.

Hjort, N.L. (1986b). Statistical Symbol Recognition. Research Monograph, Norwegian Computing Centre, Oslo.

Hjort, N.L. (1986c). Contribution to the discussion of Diaconis and Freedman's 'On the consistency of Bayes estimates'. Ann. Statist. 14, 49-55.

Hjort, N.L. (1987). Semiparametric Bayes estimators. Proceedings of the First World Congress of the Bernoulli Society, VNU Science Press.

Hjort, N.L. (1988). Contribution to the discussion of Hinkley's lectures on bootstrapping techniques. To appear in Scand. J. Statist.

Hjort, N.L. (1990a). Goodness of fit tests in models for life history data based on cumulative hazard rates. Ann. Statist. 18, 1221-1258.

Hjort, N.L. (1990b). Nonparametric Bayes estimators based on Beta processes in models for life history data. Ann. Statist. 14, 1259-1294.

Hjort, N.L. (1991a). On inference in parametric survival data models. Int. Statist. Review, to appear.

Hjort, N.L. (1991b). Bayesian and empirical Bayesian bootstrapping. Paper presented at the Fourth València meeting on Bayesian Statistics. Statistical Research Report, Department of Mathematics, University of Oslo.

Hjort, N.L. (1991c). Estimation in moderately misspecified models. Statistical Research Report, Department of Mathematics, University of Oslo.

Olkin, I. and Spiegelman, C.H. (1987). A semiparametric approach to density estimation. J. Amer. Statist. Assoc. 82, 858-865.

Ramlau-Hansen, H. (1983). Smoothing counting process intensities by means of kernel functions. Ann. Statist. 11, 453-466.

Tanner, M.A. and Wong, W.H. (1983). The estimation of the hazard function from randomly censored data. Ann. Statist. 11, 989-993.

Yandell, B.S. (1983). Nonparametric inference for rates and densities with censored serial data. Ann. Statist. 11, 1119-1135. 